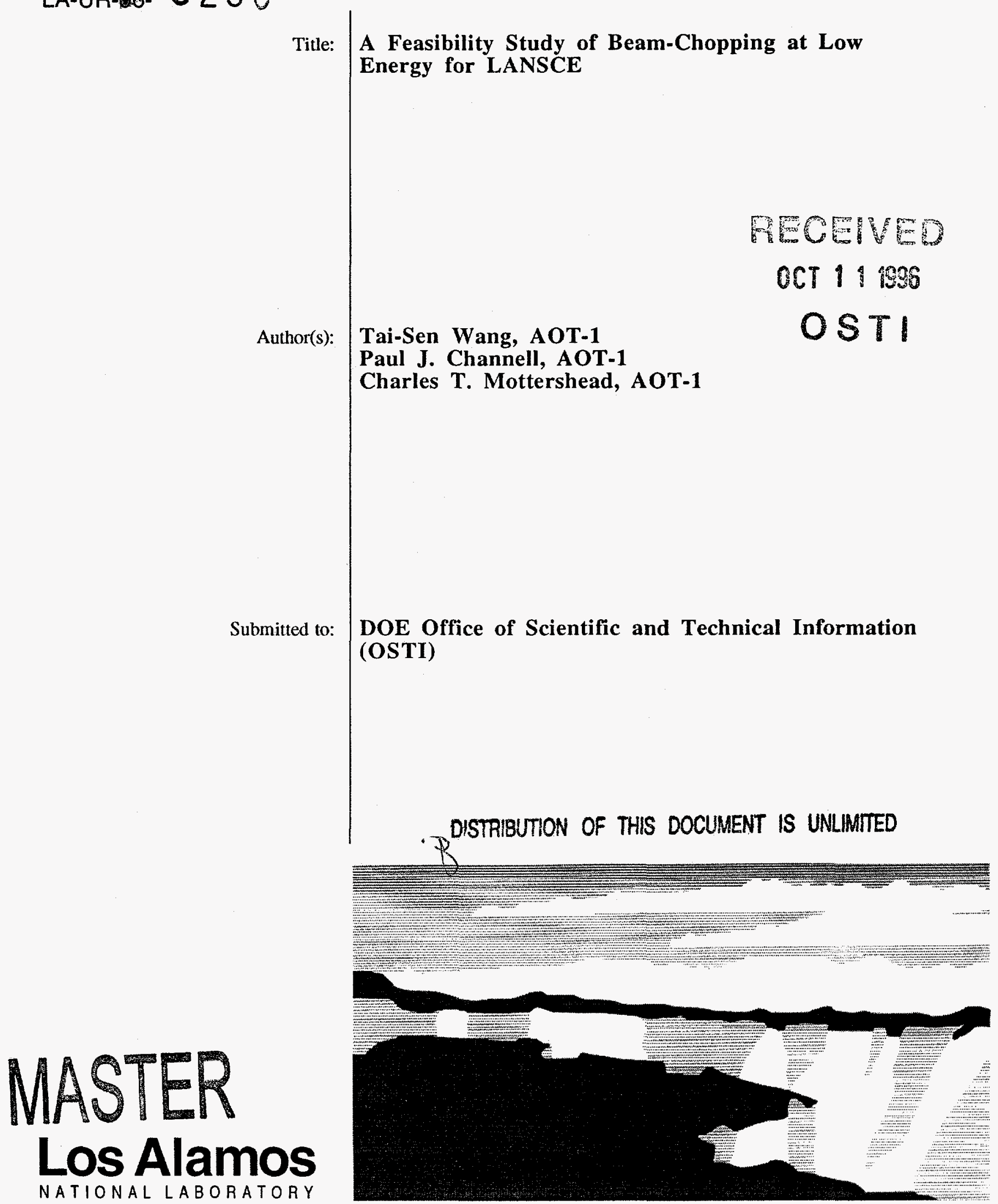

Los Alamos National Laboratory, an affirmative action/equal opportunity employer, is operated by the University of California for the U.S. Department of Energy under contract W-7405-ENG-36. By acceptance of this article, the publisher recognizes that the U.S. Government retains a nonexclusive, royaltyfree license to publish or reproduce the published form of this contribution, or to allow others to do so, for U.S. Government purposes. The Los Alamos National Laboratory requests that the publisher identify this article as work performed under the auspices of the U.S. Department of Energy. 


\section{DISCLAIMER}

Portions of this document may be illegible in electronic image products. Images are produced from the best available original document. 


\title{
A Feasibility Study of Beam-Chopping at Low Energy for LANSCE
}

Tai-Sen Wang*, Paul Channell, and Charles T. Mottershead

\begin{abstract}
This is the final report of a one-year, Laboratory-Directed Research and Development (LDRD) project at the Los Alamos National Laboratory (LANL). If a beam-chopping system could be developed for the Los Alamos Meson Physics Facility low-energy beam line, there would be potential to operate the Los Alamos Neutron Scattering Center (LANSCE) at much higher power and duty factor and enable such operation with a radio-frequency quadrapole (RFQ) injector. This would greatly extend the capability of the facility. To accommodate LANSCE operation in the new configuration, a chopped beam must be created in the low-energy transport line before the RFQ. Chopping in this region has never been demonstrated and constitutes the major uncertainty of the proposal and determines the critical path for project completion. This study produces a better understanding of the physics involved in chopping an $\mathrm{H}^{-}$ beam in a dilute plasma background, and in transporting a chopped $\mathrm{H}^{-}$beam through a neutralized or partially neutralized plasma channel, as well as an estimate for the optimum neutralization strategy for the beam chopping and transport between the ion source and the RFQ.
\end{abstract}

\section{Background and Research Objectives}

A study is underway to establish the accelerator configuration for the proposed LongPulse Spallation Source (LPSS). The LPSS will utilize the Los Alamos Meson Physics Facility (LAMPF) linac to provide over $1 \mathrm{MW}$ of proton-beam power to the spallation-neutron target at a pulse-repetition rate of $60 \mathrm{~Hz}$. This power and duty factor implies operation of the linac at unprecedented peak currents that cannot be supplied by the present linac configuration. The optimal solution found by the study utilizes a radio-frequency-quadrupole accelerator (RFQ) to replace the injector, low-energy, beam-transport line (LEBT), and first tank of the linac. High operating efficiency and low beam losses are expected with this replacement.

* Principal investigator, e-mail: twang@lanl.gov 
Replacement of the LEBT, however, requires replacement of the chopping function that provides the appropriately time-structured $\mathrm{H}^{-}$beam to the Los Alamos Neutron Scattering Center (LANSCE) storage ring. Chopping (removal of $25 \%$ of the beam at a $2.8 \mathrm{MHz}$ rate) is currently accomplished by a fast deflector device in the LEBT. With the new configuration, chopping is best accomplished before injection into the RFQ, at a low energy of $100 \mathrm{keV}$ as opposed to the 750-keV energy of the present LEBT. The problem of chopping can be complicated by the neutralization of the beam by background gas $[1,2]$. Chopping the beam causes disruption of the neutralization uniformity and subsequent beam-phase-space distortion. An attempt to implement such chopping [3] at Brookhaven National Laboratory failed for this reason. However, substantially higher beam currents and lower energies were used. At the limit of high energy and low current, the chopping will clearly work. Additionally, a twostream plasma instability that will disrupt the beam can arise under these circumstances.

The initial goal of this study was to evaluate the currently adopted chopping scheme. The intent was to look for possible improvement and modification to the present design or, if necessary, to propose a new design for the chopper. Another objective of this study was to check and understand beam neutralization, beam transport, and dynamics in the chopper. This study yielded useful results not only directly applicable to the design of the LPSS LEBT section but also useful for designing a wide class of $\mathrm{H}^{-}$LEBT sections.

\section{Importance to LANL's Science and Technology Base and National R\&D Needs}

This project supports Los Alamos core competencies in nuclear science, plasmas, and beams as well as complex experimentation and measurement. The LPSS project is an important part of the LANL neutron laboratory tactical goal, providing a substantial facility to the Laboratory. While most of the projected linac changes involve standard technology with assured success, the chopping remains an unproven and relatively uncertain aspect. Resolution of this problem must be done before a final construction plan is formulated. The alternatives to chopping before the RFQ are far more expensive and require substantial configuration changes. Although the problem of chopping at low energies has excited great interest, a concerted study has never been attempted. Results of such a study will form a vital contribution to accelerator physics. 


\section{Scientific Approach and Results to Date}

The study proceeded by estimating the beam neutralization in the chopper region based on the estimated gas pressure. We found that at the LPSS-beam-parameter range, it takes less than $200 \mu \mathrm{s}$ for the beam to become fully neutralized in the chopper. This result is consistent with the observations made at LAMPF. We then tried to understand how the beam phase space can be affected by the plasma fluctuations caused by the applied sweeping field. We made a simple model in which the perturbing field due to the ions is approximated by the field of an oscillating ion column of uniform distribution with a sharp boundary. The evolution of the beam phase space is then studied by tracing a slice of the beam through the chopper. A computer program using the particle-in-cell (PIC) algorithm was coded for this purpose. A typical result is shown in Figure 1, where the phase spaces of a beam slice at the end of a 70$\mathrm{cm}$ long chopper having a displaced ion column is compared with the case of zero ion displacement. The degradation of beam emittance caused by the displaced ions is evident in this example. We concluded that at $100-\mathrm{keV}$ particle energy, the phase-space distortion due to the fluctuation in the background plasma may he intolerable if the same type of chopper now in LAMPF were used in LPSS. This result suggests that one should pay attention to the effect due to the fluctuation in beam neutralization when transporting a chopping beam with periodically varying intensity through a plasma background. To avoid the plasma build up and excessive beam neutralization, one can consider transporting a chopped beam by using electrostatic focusing elements with strong enough field to clear the ions created in the beam channel.

More effort was spent on looking for a new beam chopping scheme and on designing a beam line with electrostatic focusing elements that would minimize beam neutralization. The elaboration led to the invention of a new type of deflector, the quadrupole slow-wave deflector (QSWD) and a conceptual design of the beam transport from the ion source to the RFQ in the LPSS. During the course of this study, measurement results and operational experience of the planar coaxial-plate deflector now in service at LAMPF were consulted for estimations. Analytical calculations using a conformal mapping technique have been performed to compute the static electric field in a deflector with special geometry. An envelope tracking program QKICKE and a PIC simulation code QKICKP were developed by using the analytically calculated electric field to study the motion of the $\mathrm{H}^{-}$beam in the deflector. The programs TRACE2D and KTRACE were used to design the optics of the beam matching from ion source to the RFQ. A technical note describing the idea of a QSWD and a conceptual design of the chopper in LPSS has been prepared [4]. 
The idea of a QSWD is to modify one pair of the poles in an electrostatic quadrupole and to use them as the deflector electrodes. Figure 2 shows the structure of a QSWD, in which the horizontal electrodes are the same as those in a normal quadrupole and the vertical electrodes are made of hyperbolically curved parallel plates connected through the coaxial cables behind the ground plate to form a helical-type, slow-wave structure. During operation, a dc voltage $\mathrm{V}$ is constantly applied to the horizontal electrodes. The vertical electrodes are connected to pulse-power sources, which establish the beam-deflection field when the two slow-wave structures are excited separately by synchronized pulses of voltages $\mathrm{V}$ and $-\mathrm{V}$. When the pulsing voltages are switched off, the QSWD functions as an electrostatic quadrupole focusing the beam in the vertical direction. This constant quadrupole electric field is also used to sweep off the ions and electrons produced by the beam-gas scattering. A QSWD therefore is one simple structure that performs ion clearing and linear focusing in the transmit mode and also serves as a fast kicker in the deflection mode. Utilizing a QSWD for beam-chopping can avoid all the complicated beam-plasma effects that may occur in a neutralized beam. The beam chopping can be accomplished by inserting an aperture limiter to the downstream beam line to stop the deflected beam. Note that, instead of focusing the beam to a small spot, the quadrupole field focuses the beam in the vertical direction and spreads it horizontally on the aperture limiter, so the cooling of the aperture limiter can be handled easily.

Using the idea of a QSWD, we have made a conceptual design of an $\mathrm{H}^{-}$chopper for LPSS and the optics of the transport line from the source to the RFQ. This design is based on the following given conditions at the ion source: a $100-\mathrm{keV}, 15-\mathrm{mA}$ beam with a normalized rms emittance of $0.02 \pi \mathrm{cm}$ mrad. The initial beam envelope has a round cross section with $0.5-\mathrm{cm}$ radius and a divergence of $65 \mathrm{mrad}$. The beam is focused into a QSWD by an $18-\mathrm{cm}$ long solenoid of 0.5 -T field placed at $20 \mathrm{~cm}$ in front of the source. The QSWD has a length of $70 \mathrm{~cm}$ and a pole-tip to pole-tip aperture of $7.0 \mathrm{~cm}$. At the nominal operation voltage of $0.7 \mathrm{kV}$ on both the quadrupole and the deflector, an undeflected beam will have a $1-\mathrm{cm}$ waist at the end of the structure, and a deflected beam can be separated from the undeflected one by $0.8 \mathrm{~cm}$, edge-to-edge, at exit. A 1-cm limiting aperture is established at $9 \mathrm{~cm}$ downstream from the QSWD to stop the deflected beam. Figure 3 shows a TRACE2D output for the layout of our design including the beam-transport from the ion source to the RFQ using three electrostatic quadrupoles and one magnetic solenoid for beam matching. The magnetic bending dipole is inserted for the purpose of merging the $\mathrm{H}^{-}$and the proton beamlines before the RFQ. Due to the finite mobility of ions, a small amount of neutralization is unavoidable. A crude estimate shows that a vacuum of at least $10^{-5}$ Torr is needed in the QSWD for the chopper to operate successfully. At this pressure, the beam neutralization in the chopper is about a few percent. 
We note that although our design is aimed at the application to LPSS, the underlying principle of a QSWD should be applicable to all kinds of charged-particle beams. Also, the computer programs developed in this project can be used to explore a wide range of problems involving beam propagation in a neutralized environment and to design QSWDs for any possible applications.

\section{References}

1. Stevens, R., "Traveling Wave Choppers for LANSCE II," in Proceedings of the Workshop on Ion Source Issue Relevant to a Pulsed Spallation Neutron Source, Part 2, Lawrence Berkeley Laboratory, Oct. 24-26, 1994.

2. Pozimski, J., "LEBT: Ion Source to RFQ, Advantages and Problems of Space Charge Compensation," in Proceedings of the Workshop on Ion Source Issue Relevant to a Pulsed Spallation Neutron Source, Part 2, Lawrence Berkeley Laboratory, Oct. 24-26, 1994.

3. Alessi, J., "Fast Beam Chopping at BNL," in Proceedings of the Workshop on Ion Source Issue Relevant to a Pulsed Spallation Neutron Source, Part 2, Lawrence Berkeley Laboratory, Oct. 24-26, 1994.

4. Wang, T., et al., "Quadrupole Slow-Wave Deflector: a Novel Idea for Chopping Charged-Particle Beams," Los Alamos National Laboratory AOT Division Technical Note, LA-CP-95-281, Oct. 23, 1995. 


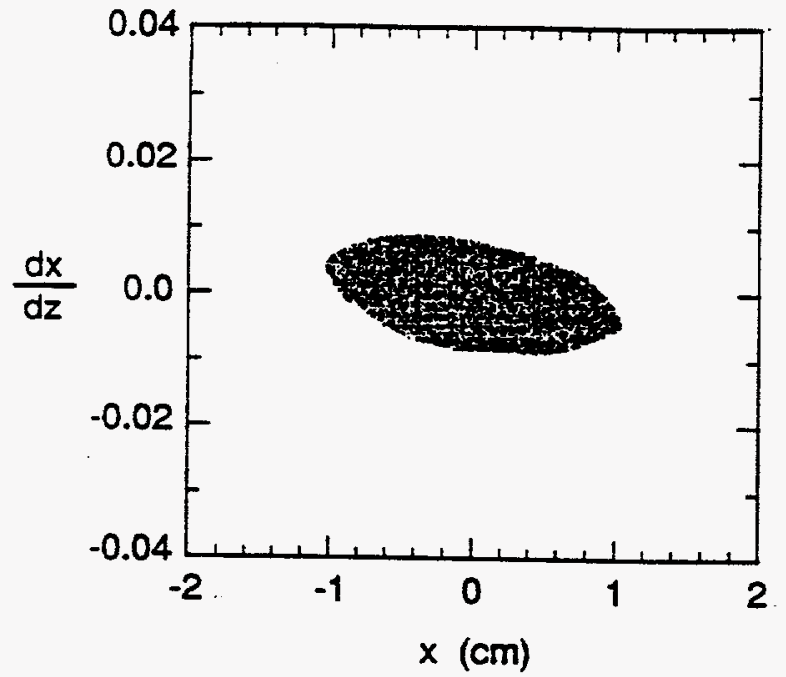

(a)

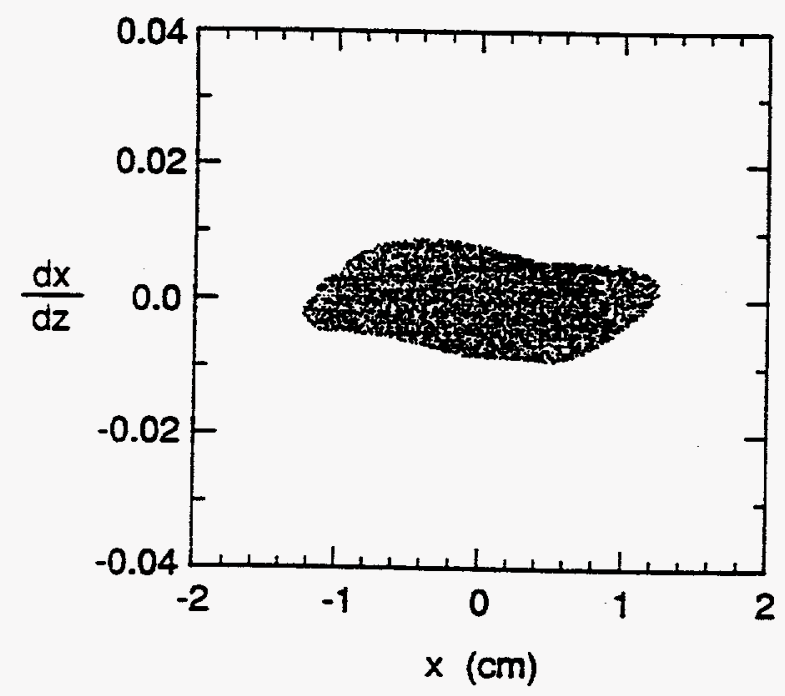

(c)

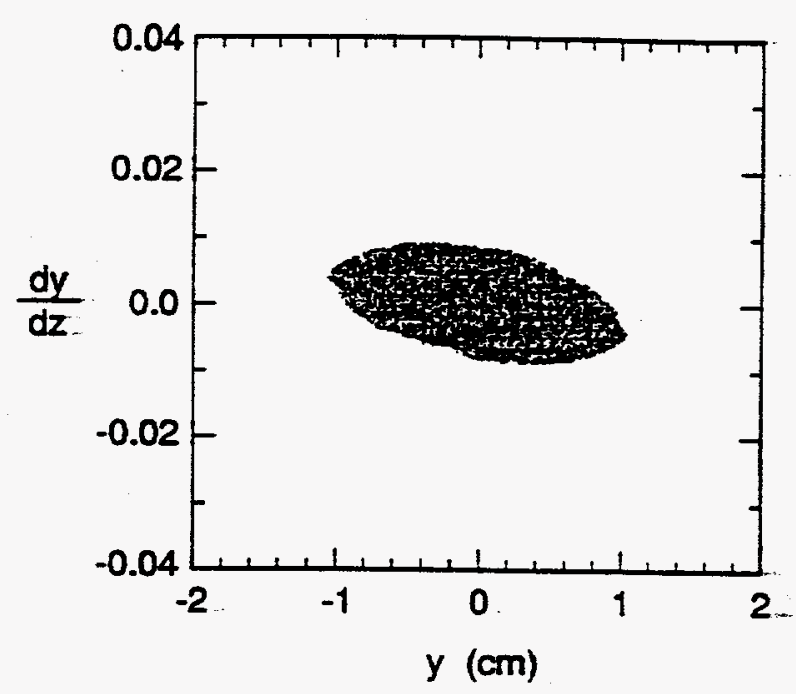

(b)

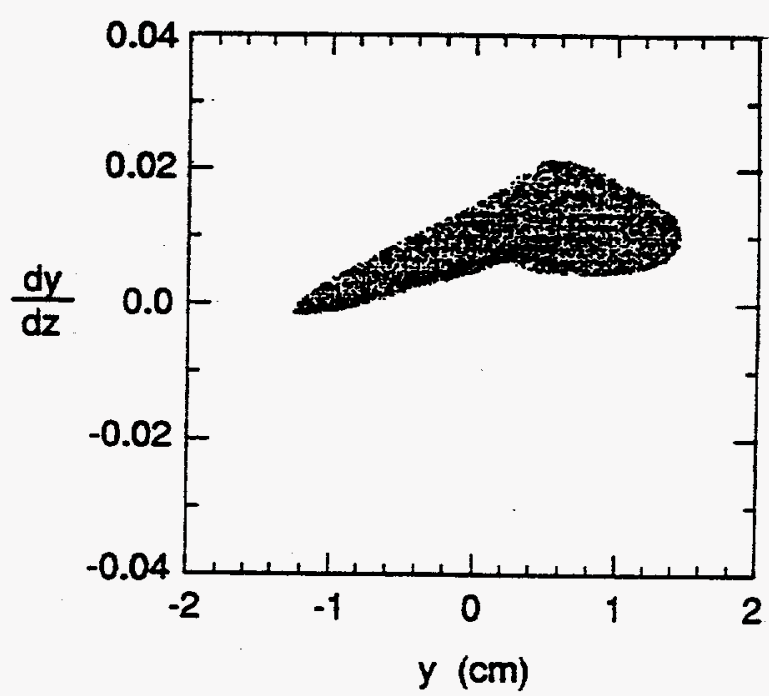

(d)

Figure 1. The phase space of an $\mathrm{H}$-minus beam slice at the end of a $70-\mathrm{cm}$ long transport line with a proton background that fully neutralizes the beam. (a) and (b): protons are uniformly distributed within the beam channel. (c) and (d): protons are displaced from the beam in the $y$-direction by approximately $0.6 \mathrm{~cm}$. 


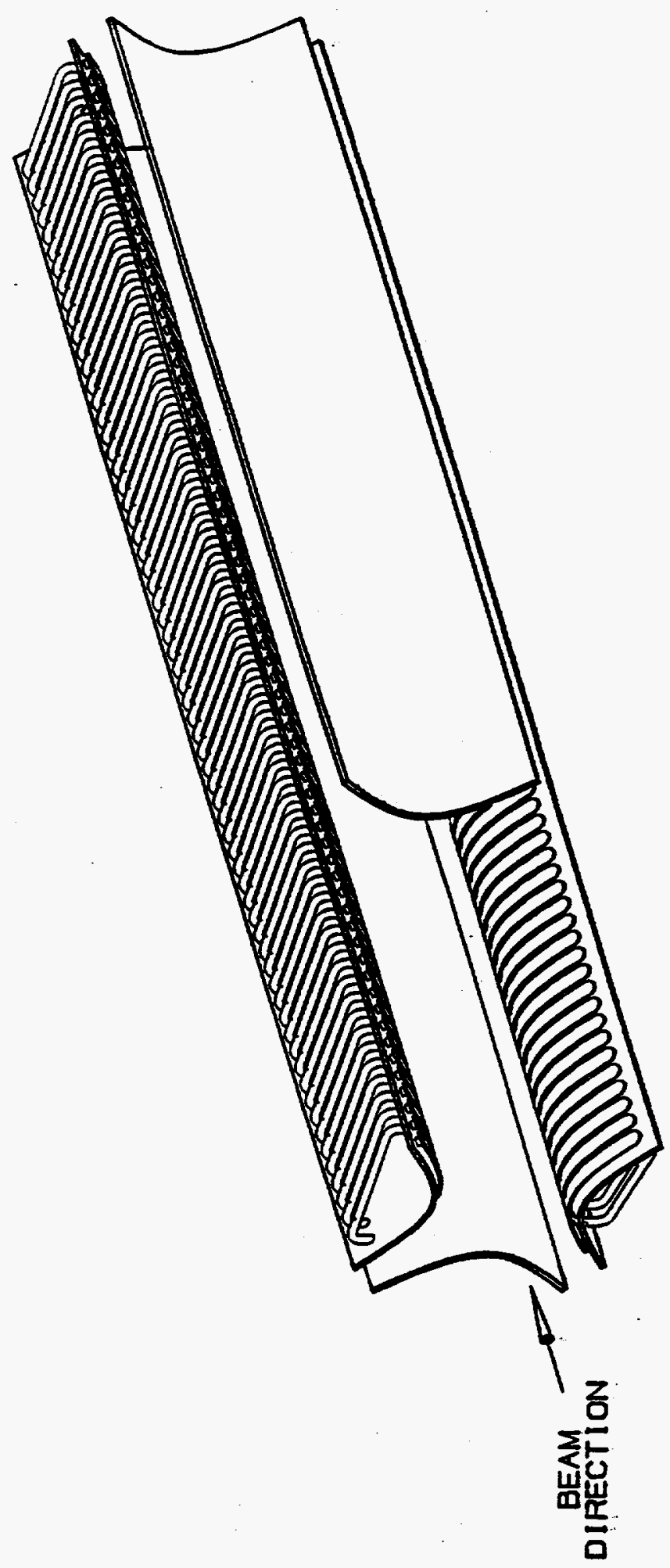

Figure 2. An illustration of the structure of a quadrupole slow-wave deflector (QSWD). 

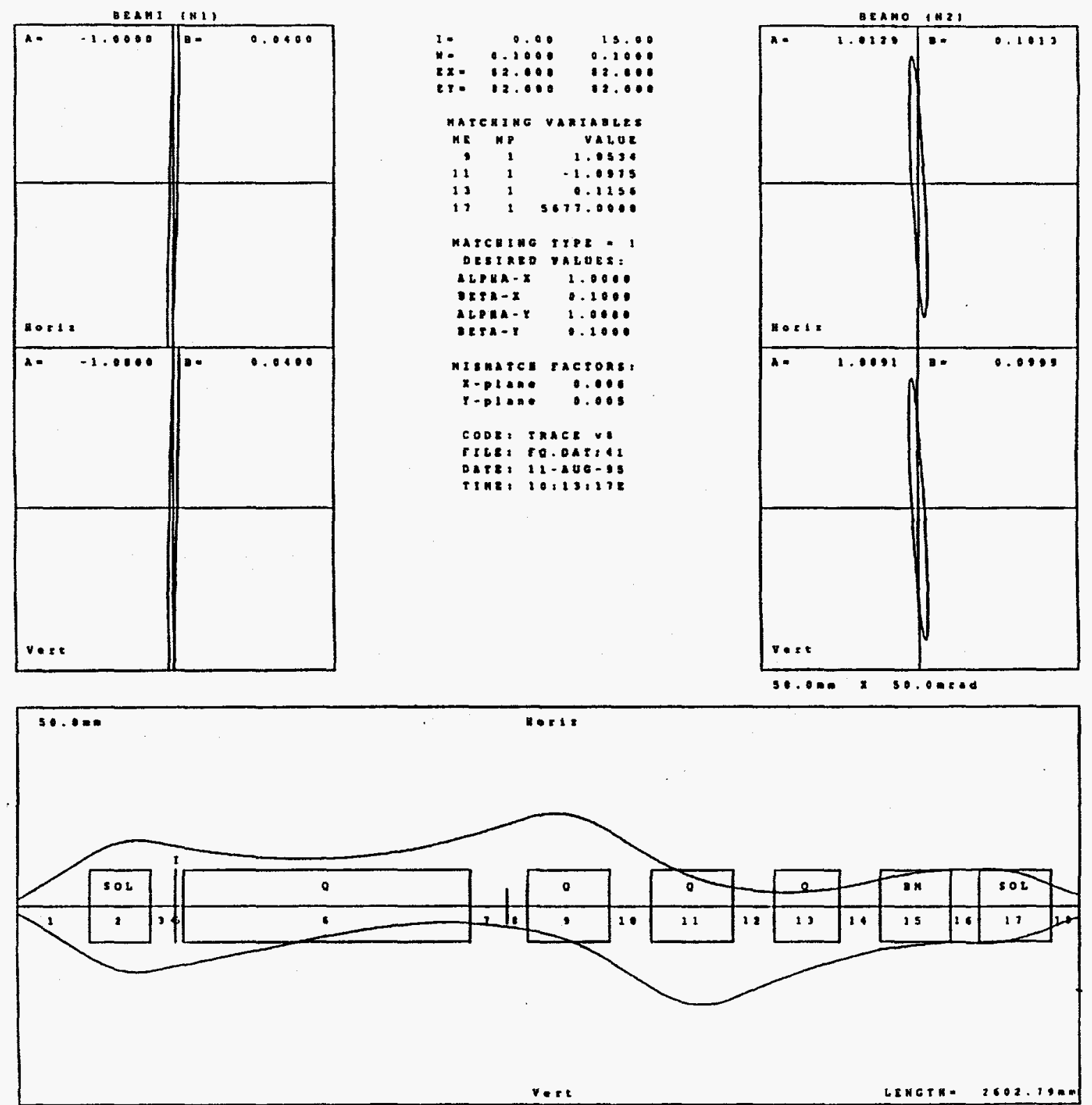

Figure 3. The output from a TRACE2D run showing the beam envelopes, the initial and the final beam phase spaces, and the optical elements from the ion source to the RFQ in a conceptual design for the LPSS. 